\title{
On the Fate of Adenovirus DNA in KB Cells
}

Electron microscopic and biochemical studies on the process of infection by adenoviruses have shown that after penetration into the cell the virions lose the pentons and are then gradually uncoated $(1,2)$. Later adenovirus DNA is found in the cell nucleus, where DNA replication takes place. The mechanism of replication of linear adenovirus DNA molecules is still unknown. This report presents the first results of a study on the mechanism of adenovirus DNA replication and concerns especially the fate of the parental DNA molecules. To investigate whether tumorigenic and nontumorigenic adenovirus types differ in this aspect of the infection process, experiments were performed with adenovirus type 5 (Ad5) and type 12 (Ad12). As only parental molecules in the cell nucleus are important for replication, nuclei were prepared from infected cells and the parental viral DNA associated with them was examined.

KB cells were grown in monolayer cultures in Farle's balanced salt medium (Flow, Irvine, Scotland) containing $0.5 \%$ lactalbumin, $5 \%$ calf serum (Flow, Irvine, Scotland), $100 \mathrm{C} / \mathrm{ml}$ of penicillin $\mathrm{G}$ and $100 \mu \mathrm{g} / \mathrm{ml}$ of streptomycin sulfate (Mycofarm, Delft, Holland). Suspension cultures were obtained by trypsinization of monolayers and addition of the cells to the above medium. Purified virus preparations of adenovirus type 5 (ATCC) and type 12 (Huie) were obtained according to Green and Pina (3). For the isolation of viruses containing labeled DNA, $100 \mu \mathrm{Ci}$ thymidine- $6-{ }^{3} \mathrm{H}$ were added to $3 \times 10^{7}$ infected cells. Suspension cultures in stoppered flasks were inoculated with labeled or nonlabeled virus pools $\left(2 \times 10^{6}\right.$ cells and $3-4 \times$ $10^{10}$ virions per milliliter). At several times p.i. cells were sedimented and washed after which the infected nuclei were isolated according to Penman (4), without detergent treatment. The associated viral DNA was isolated (5) and analyzed by centrifugation as described in the legend of Fig. 1. Fractions were counted in a liquid scintillation counter. The formation of newly synthesized viral DNA was studied with nonradioactive virus preparations under the conditions mentioned above with the addition of $i \mu \mathrm{Ci} / \mathrm{ml}$ thrmidine- $6-{ }^{3} \mathrm{H}(10 \mathrm{Ci} / \mathrm{mmole})$ and $4 \mu \mathrm{g} / \mathrm{ml}$ mitomycin C (Sigma, St. Louis, Mo).

Double-diffusion experiments were performed with rabbit antiserum prepared against Ad5 and Ad12 (Grand Island Biological Co., Grand Island, NY) in $0.01 \mathrm{M}$ veronal buffer, $\mathrm{pH}$ 8.4. Purified radioactive Ad5 and Ad12 DNA was prepared according to van der $\mathrm{Eb}$, van Kesteren, and van Bruggen (6) with pronase and used as a marker in the centrifugation experiments. The purified DNA preparations of Ad5 and Ad12 contained only $31 \mathrm{~S}$ and $30 \mathrm{~S}$ molecules, respectively.

$\mathrm{KB}$ cells infected with radioactive $\mathrm{Ad} 5$ were isolated at different times p.i. Purified cell nuclei were prepared and the viral DNA in the nuclei was analyzed. Radioactivity measurements showed that under our condi tions the amount of parental viral DNA in the nucleus did not increase after 5 hours of incubation. Calculations revealed that about 5000 molecules/nucleus were present. Analysis of Ad5 DNA showed that until 18 hours p.i. the parental molecules had the same sedimentation coefficient as DNA isolated from purified virions by phenol extraction (Fig. 1). No measurable amount of faster or slower sedimenting molecules could be de. tected.

Similar experiments with type 12 showed that 5 hours p.i. normal linear molecules $(30 \mathrm{~S})$ were found in the nucleus (Fig. 2). However, 9 hours p.i. a $20 \mathrm{~S}$ component could be detected and 18 hours p.i. an extensive degradation of the parental molecules had taken place. In some experiments $13 \mathrm{~S}$ fragments were also observed. The $1 \mathrm{t}$. hour samples of Ad5 and Ad12 DNA were analyzed by $\mathrm{CsCl}$ equilibrium density gradient centrifugation in the presence of eth- 


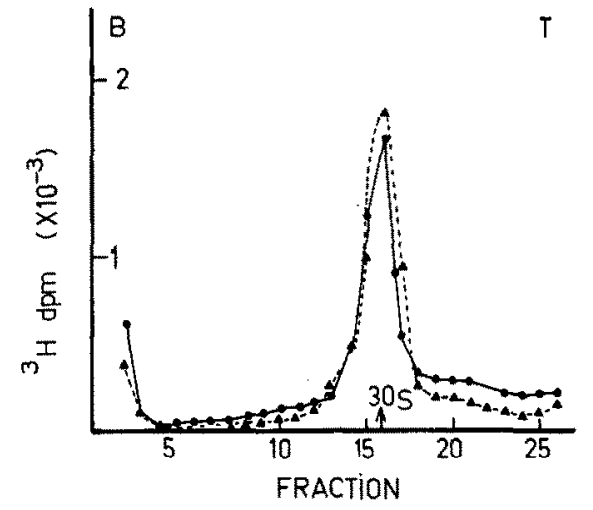

FIG. 1. Sucrose gradient centrifugation in 520\% suerose, $0.01 M$ Tris, pH 8.1, $0.001 M$ EDTA, and $0.1 \%$ sodium dodecylsulfate of viral DNA, isolated from nuclei of $\mathrm{KB}$ cells infected with Ad5 virions containing ${ }^{3} \mathrm{H}-\mathrm{DNA}$. Centrifugation was performed for 6 hours at $24,000 \mathrm{rpm}$ at $15^{\circ}$ in the Spinco SW25 rotor. Viral DNA was isolated 5 hours $(\boldsymbol{\Delta})$ and 18 hours $(\mathbf{O})$ p.i. The position of ${ }^{14}$ C-labeled Ad12 DNA (30S) is marked by an arrow.

idium bromide $(120 \mu \mathrm{g} / \mathrm{ml})$ for 50 hours at $38,000 \mathrm{rpm}$ in the Spinco R50 rotor. Molecules with densities of covalently closed circular molecules could not be observed.

The cause of the degradation of Ad12 parental DNA might be the endonuclease activity found in the pentons of purified Ad12 virions ( 7$)$. Experiments were performed to identify an endonuclease activity in virions of both types. Ad5 and Ad12 virus preparations in Tris $0.01 M, \mathrm{pH} 8.0$, and $\mathrm{NaCl} 0.15 M$ were stored in the presence of purified ${ }^{3} \mathrm{H}$-labeled Ad5 and Ad12 DNA, respectively. During storage viral capsomers were released and DNA degradation could occur.

After 6 days of incubation almost no intact virions were found by $\mathrm{CsCl}$ density centrifugation and an extensive breakdown of the virions was detected by double-diffusion precipitation. The incubation samples were analyzed by sucrose gradient centrifugation. Control experiments were performed in absence of viral protein.

Analysis of labeled DNA showed that under these circumstances Ad12 DNA was almost completely converted into $20 \mathrm{~S}$ fragments while in the Ad5 system no degradation could be detected.

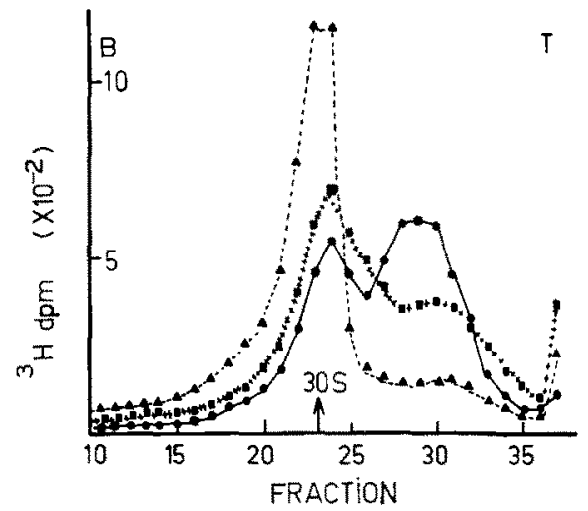

Fig. 2. Sucrose gradient centrifugation in 5$20 \%$ sucrose, $0.01 M$ Tris pH 8.1, $0.001 M$ EDTA, and $0.1 \%$ sodium dodecylsulfate of viral DNA isolated from nuclei of $\mathrm{KB}$ cells infected with Ad12 virions containing ${ }^{3} \mathrm{H}-\mathrm{DNA}$. Centrifugation was performed for 6 hours at $24,000 \mathrm{rpm}$ at $15^{\circ}$ in the Spinco SW25 rotor. Viral DNA was isolated $5(\mathbf{A}), 9(\mathbf{O})$, and 18 hours (C) p.i. The position of ${ }^{14}$ C-labeled Ad12 DNA (30S) is marked by an arrow.

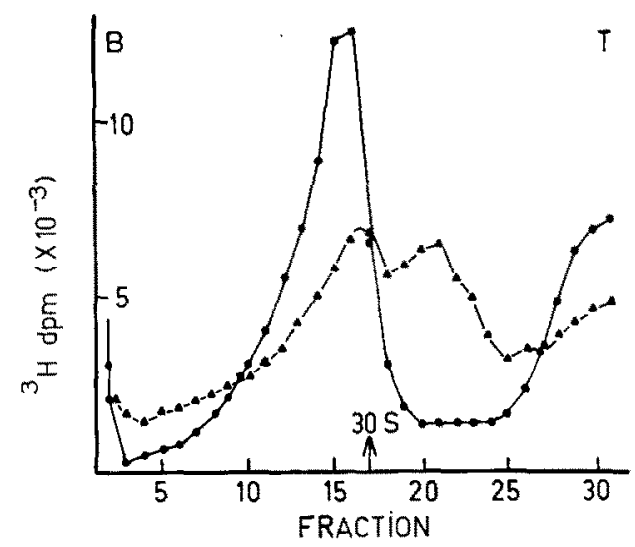

Fig. 3. Sucrose gradient centrifugation in $5-$ $20 \%$ sucrose, $0.01 M$ Tris, pH 8.1, $0.001 M$ EDTA, and $0.1 \%$ sodium dodecylsulfate of new viral DNA, synthesized 15-18 hours p.i. in nuclei of infected $\mathrm{KB}$ cells. Centrifugation was performed for 6 hours at $24,000 \mathrm{rpm}$ at $15^{\circ}$ in the Spinco SW25 rotor. Cells were infected with nonradioactive virus preparations of $\mathrm{Ad5}(\boldsymbol{C})$ or $\mathrm{Ad} 12$ (A). The position of ${ }^{14} \mathrm{C}$-labeled Ad12 DNA (30S) is marked by an arrow.

These observations make it very likely that breakdown of Ad12 DNA is performed by the endonuclease activity in Ad12 virions. The absence of such an activity in Ad5 


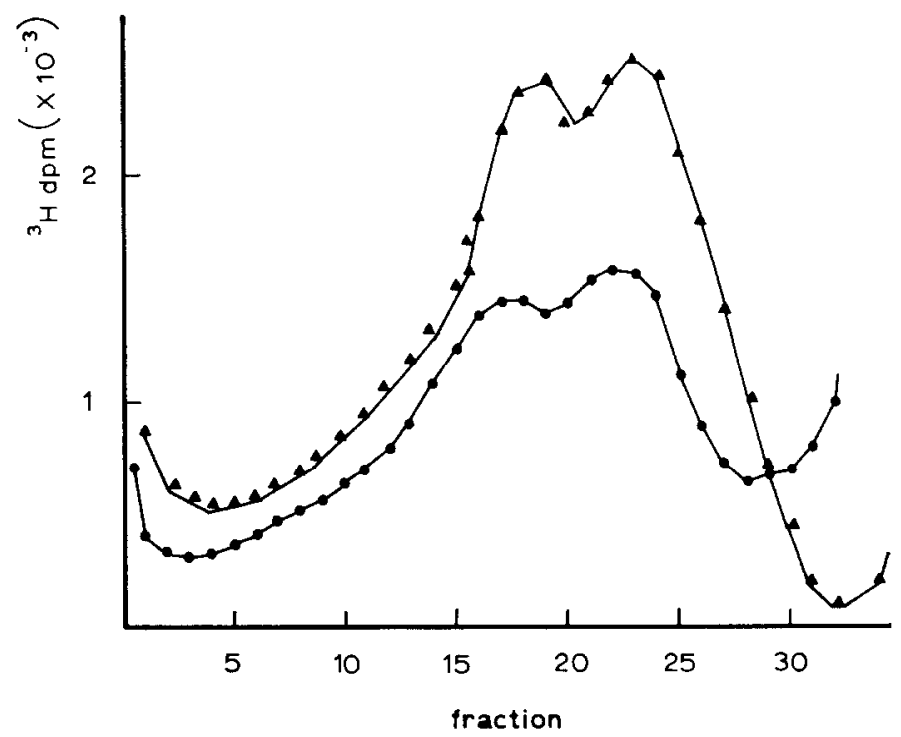

FIG. 4. Sucrose gradient centrifugation in 5-20\% sucrose, $0.01 M$ Tris, pH 8.1, $0.001 M$ EDTA, and $0.1 \%$ sodium dodecylsulfate of new viral DNA, synthesized 15-18 hours p.i. in nuclei of Ad12-infeeted KB cells. DNA was isolated 18 hours p.i. from $0.6 \times 10^{5}$ cells (O) and after a chase with unlabeled thymidine until 23 hours p.i. from $10^{6}$ cells (A). Centrifugation was performed for 6 hours at $24,000 \mathrm{rpm}$ at $15^{\circ}$ in the Spinco $\mathrm{SW} 25$ rotor.

virions might explain the absence of degradation of Ad5 DNA in the cell nucleus. The meaning of this difference between the two types is unclear. As suggested by Doerfler (7) the fragmented state of adenovirus DNA in abortively infected cells may be related to the endonuclease activity in the virions $(8)$.

In order to investigate whether splitting of Ad12 parental molecules has an influence on the daughter molecules, newly synthesized viral DNA was investigated. Although cellular DNA synthesis is strongly inhibited late after infection, residual cellular synthesis was blocked with mitomycin $\mathrm{C}$. KB cells were infected with nonlabeled Ad5 and Ad12 virus preparations in the presence of $4 \mu \mathrm{g} / \mathrm{ml}$ mitomycin C. After addition of this drug viral DNA synthesis proceeds and virus is formed, while cellular DNA synthesis is inhibited by $99 \%(9,10$, and own results). Under our conditions Ad5 and Ad12 DNA synthesis started at about 14 hours p.i. Newly synthesized DNA was labeled by addition of ${ }^{3} \mathrm{H}$-thymidine to the infection medium 15-18 hours p.i. and analyzed. More than $98 \%$ of the new DNA had the buoyant density of the parental molecules (1.714 $\mathrm{g} / \mathrm{cm}^{3}$ for $\Lambda \mathrm{d} 5$ and $1.708 \mathrm{~g} / \mathrm{cm}^{3}$ for $\mathrm{Ad} 12$ ). This means that new DNA was neither of cellular origin nor in a single-stranded form.

After infection with Ad5, sucrose gradient centrifugation of new DNA showed only $31 \mathrm{~S}$ molecules. Infection with Ad12 resulted in the presence of 30 and $20 \mathrm{~S}$ molecules (Fig. 3 ). The distribution was almost identical to that obtained for parental molecules at 18 hours p.i.

The $20 \mathrm{~S}$ fraction of new Ad12 DNA might originate from replication of $20 \mathrm{~S}$ parental molecules or from the action of Ad12 endonuclease on new $30 \mathrm{~S}$ molecules. Since the observed rate of degradation of parental molecules was rather low and the time of exposure of new DNA to endonuclease activity was about four times shorter than that of parental DNA, the second possibility is rather unlikely. Moreover, the amount of new DNA was estimated to be about 3-10 times higher than the amount of parental molecules which also does not favour degradation.

To obtain morc information on the origin of $20 \mathrm{~S}$ molecules a pulse-chase experiment was performed. $\mathrm{KB}$ cells were infected with Ad12 virions and newly synthesized DNA 
was labeled with ${ }^{3} \mathrm{H}$-thymidine $15-18$ hours p.i. At 18 hours p.i. a sample was taken and the associated DNA extracted, while the remainder of the cells was washed and further incubated in fresh medium containing $10^{-5}$ $M$ thymidine. At 23 hours p.i. DNA was extracted and analyzed by sucrose-gradient centrifugation together with the 18-hour sample (Fig. 4). During the chase the amount of labeled DNA did not increase, while the distribution of labeled 30 and $20 \mathrm{~S}$ molecules did not change.

From this experiment it may be concluded that $20 \mathrm{~S}$ molecules are probably not formed by degradation of $30 \mathrm{~S}$ molecules. The absence of degradation during the chase cannot be explained by a dilution effect caused by new unlabeled molecules resulting in a strongly decreased rate of degradation of labeled molecules since DNA formed between 20 and 23 hours p.i. is also mainly in the fragmented form (11 and own observations). If these 23-hour fragments should originate by endonuclease action, one should expect that $30 \mathrm{~S}$ molecules formed 15-18 hours p.i. should be degraded as well. The possibility that $30 \mathrm{~S}$ molecules have a chance to be degraded only if attached to the replication site cannot be excluded but is not very likely. Although these experiments are not fully conclusive, they suggest that Ad12 fragments are able to replicate. This would make a mechanism of DNA replication involving circular replicating molecules un- likely. The absence of covalently closed circular molecules, as determined in ethidium bromide density centrifugation, is in accordance with this view. An extended study on replicating adenovirus DNA is in progress.

\section{ACKNOWLEDGMENTS}

The author thanks Prof. Dr. H. S. Jansz for his interest and Miss A.Th. Mulder and Miss M. G. Kuijk for their able technical assistance.

\section{REFERENCES}

1. Sussendach, J. S., Virology 33, 567-574 (1967).

2. Chardonnet, Y., and Dales, S., Virology 40, 462-477 (1970).

3. Green, M., and Pina, M., Virology 20, 199207 (1963).

4. Penman, S., J. Mol. Biol. 17, 117-130 (1966).

5. Hrrt, B., J. Mol. Biol. 26, 365-369 (1967).

6. van der Eb, A. J., van Kesteren, L. W., and van Bruggen, E. F. J. Biochim. Biophys. Acta 182, 530-541 (1969).

7. DOeRfler, W., J. Virol. 6, 652-666 (1970).

8. Burlingham, B. T., and Doerfler, W., Fed. Proc. 28, 434 (1969).

9. Ginsberg, H. S., Cold Spring Harbor Symp. Quant. Biol. 27, 234 (1962).

10. Magee, W. E., and Mrluer, O. V., Biochim. Biophys. Acta 55, 818 (1962).

11. Domrfler, W., Virology 38, 587-606 (1969).

Laboratory for Physiological

J. S. Sussenbach

Chemistry

State University of Utrecht

Utrecht, Netherlands

Accepted September 12, 1971 